\title{
THE DISTRIBUTION OF SELF-STERILITY ALLELES IN POPULATIONS
}

\author{
SEWALL WRIGHT \\ The University of Chicago, Chicago, Illinois
}

Received February I8, 1939

HE phenomenon of self-sterility is known to be very common among
higher plants. The genetic mechanism in most cases studied seems to be that discovered by EAST and MANGELSDORF in Nicotiana alata and $N$. Sanderae. A single series of alleles, $S_{1}, S_{2} \ldots$, is involved. The growth of a pollen tube is so much inhibited in a style whose cells contain the same allele that it does not reach the embryo sac. It is obvious that all individuals must be heterozygous and that a population cannot persist with less than $3 S$-alleles. It is also fairly obvious that selection would tend to increase the frequency of any additional alleles that may appear. DR. Sterling Emerson has been investigating a series of at least 37 selfsterility alleles of this sort, found in Oenothera organensis, a species in which the entire population apparently consists of less than one thousand individuals, scattered in small groups among certain canyons of the Organ Mountains of New Mexico (Emerson 1938, I939). I am indebted to him for calling my attention to the interesting questions involved in the effects of selection and inbreeding in this case.

\section{SELECTION PRESSURE}

We assume the existence of a series of $n$ self-sterility alleles, $S_{1}, S_{2} \cdots S_{n}$, with frequencies $q_{1}, q_{2} \cdots q_{n}$ such that $\Sigma q=\mathrm{I}$ in a population of $N$ diploid individuals. The frequencies of zygotes containing one of these $\left(S_{i}\right)$ must be $2 q$ (with the appropriate subscript) since all are heterozygotes. The frequency of functioning $S_{i}$ female gametes is $q$, assuming no differential selection. The frequency of functioning $S_{i}$ pollen grains is not in general the same. $S_{i}$ pollen has, by hypothesis, no chance of functioning in the styles of zygotes containing $S_{i}$, but has a better than average chance in zygotes that lack $S_{i}$ (frequency I-2q) since each zygote of this class inhibits pollen of two of the other kinds. Assume that on non- $S_{i}$ styles, the ratio of successful $S_{i}$ pollen grains to successful ones of other types is as $q: R(\mathrm{I}-q)$. The total frequency of functioning $S_{i}$ pollen is then $q(\mathrm{r}-2 q) /[q+R(\mathrm{r}-q)]$. The average frequency of functioning $S_{i}$ gametes is $q(\mathrm{I}-q)(\mathrm{I}+R)$ $/ 2[q+R(\mathrm{I}-q)]$ and the change from the previous generations is therefore

$$
\Delta q=\frac{q(\mathrm{I}-q)(\mathrm{1}+R)}{2[q+R(\mathrm{I}-q)]}-q=\frac{q}{2}\left[\frac{(\mathrm{I}-R)-q(3-R)}{R+q(\mathrm{I}-R)}\right] .
$$

Letting $\hat{q}$ represent the point at which selection has no effect $(\Delta q=0)$, 


$$
\hat{q}=\frac{\mathrm{I}-R}{3-R}, \quad R=\frac{\mathrm{I}-3 \hat{q}}{\mathrm{I}-\hat{q}}, \quad \Delta q=-\frac{q(q-\hat{q})}{\mathrm{I}-3 \hat{q}+2 \hat{q} q} .
$$

If all non- $S_{i}$ alleles have the same frequency, it is obvious that $R$ will have the value $(n-3) /(n-I)$ since each non- $S_{i}$ style permits functioning of only $n-3$ of the $n-$ I types of non- $S_{i}$ pollen grains. This leads to a formula for the frequency of functioning $S_{i}$ gametes, $q(\mathrm{I}-q)(n-2) /(n-3+2 q)$ communicated to me by Dr. EmErson for this case. But the frequencies of the various alleles may be expected to drift at random about the equilibrium point as a result of the accidents of sampling. The average value of $R$ will consequently be somewhat less than $(n-3) /(n-1)$ (unless $n=3$, $R=0$ ) since pollen grains with the more abundant alleles will be inhibited in excess. We will assume that $R$ may be treated with sufficient accuracy as constant in populations that have long been subject to the same conditions (number of individuals, mutation rate in the $S$ series, rate of cross breeding, etc.).

The fluctuating variations in $R$ due to chance variations in the relative frequencies among the non- $S_{i}$ alleles are not an important qualification but variations correlated with differences in the frequency of $S_{i}$ itself introduce a systematic error. If there are only four alleles altogether, it is obvious that the three non- $S_{i}$ alleles may be expected to be somewhat more nearly equal in frequency as a result of selection, if $S_{i}$ is rare, than if it is common; and that $R$ will consequently be negatively correlated with, $q$. The upward pressure on rare alleles being approximately $q(\mathrm{I}-R) / 2 R$ the assumption that $R$ is constant slightly overestimates the upward selection pressure on rare alleles carried by a population under given conditions. This effect rapidly decreases in importance as $n$ becomes greater than 4 .

\section{MUTATION AND MIGRATION PRESSURES}

Mutation from $S_{i}$ at the rate $u$ per generation, and to it at the rate $v$, changes gene frequency at the rate $v(\mathrm{r}-q)-u q$. Crossbreeding between the population in question and the species as a whole, such that the proportion $m$ of the gametes come from the latter, changes gene frequency at the rate $-m\left(q-q_{t}\right)$ where $q_{t}$ is the frequency of $S_{i}$ in the species as a whole. The net rate of change of gene frequency and its sampling variance are as follows:

$$
\begin{aligned}
\Delta q & =\frac{q(\mathrm{I}-q)(\mathrm{I}+R)}{2[R+q(\mathrm{I}-R)]}-\left[\mathrm{I}+u+m\left(\mathrm{I}-q_{t}\right)\right] q+\left(v+m q_{t}\right)(\mathrm{I}-q) \\
\sigma_{\Delta q}^{2} & =\frac{q(\mathrm{I}-q)}{2 N} .
\end{aligned}
$$


THE DISTRIBUTION FORMULA

The general formula for the distribution of gene frequencies determined by any systematic evolutionary pressure $\Delta q$, and random variations measured by $\sigma_{\Delta g}^{2}$, has been shown to be as follows (WRIGHT I938).

$$
\phi(q)={\frac{C e^{2}}{\sigma_{\Delta q}^{2}}}^{\int\left(\Delta q / \sigma_{\Delta q}^{2}\right)^{d q}} .
$$

Substituting the values of $\Delta q$ and $\sigma_{\Delta q}^{2}$ found for the present case we have

$$
\phi(q)=C[R+q(\mathrm{I}-R)]^{2 N(1+R) /(1-R)} q^{4 N(v+m q t)-1}(\mathrm{I}-q)^{4 N[1+u+m(1-q t)]-1} .
$$

The simplest special case is that of three alleles with negligible rates of mutation and cross breeding. In this case $R=0$ since $S_{2} S_{3}$ can be fertilized only by $S_{1}$ pollen. Thus $\hat{q}=\frac{1}{3}, \Delta q=(\mathrm{I}-3 q) / 2$.

$$
\phi(q)=\frac{\Gamma(6 N)}{\Gamma(2 N) \Gamma(4 N)} q^{2 N-1}(\mathbf{I}-q)^{4 N-1} .
$$

The mean and variance of this distribution are easily found.

$$
\begin{aligned}
\bar{q} & =\int_{0}^{1} q \phi(q) d q=\frac{1}{3} \\
\sigma_{q}^{2} & =\int_{0}^{1}(q-\bar{q})^{2} \phi(q) d q=\frac{2}{9(6 N+\mathrm{I})} .
\end{aligned}
$$

The mode is at the point at which

$$
\begin{gathered}
\frac{d \log \phi(q)}{d q}=\circ \\
\text { Mode }=\frac{2 N-\mathbf{I}}{6 N-2} .
\end{gathered}
$$

These formulae can easily be generalized to allow for mutation and crossbreeding but not for the presence of a larger number of alleles. However, if values of $N, u, v, m, q_{t}$ and $R$ are assumed, the ordinates of the distribution may be calculated as multiples of $C$. The value $C$ required to make the total frequency one can then be found after estimating the sum of all frequencies. The mean $(\bar{q})$ and other statistics of the distribution can also be calculated empirically. Having found $\bar{q}$, the number of alleles $(n)$ present under the conditions is given by the reciprocal $(\bar{q}=\Sigma q / n=\mathrm{r} / n)$. The proportion of the alleles lost in each generation by the accidents of sampling can be estimated from the frequencies of the low gene frequencies $\mathrm{I} / 2 N, 2 / 2 N \cdots$, by use of the Poisson series, a question discussed in the next section. At equilibrium, losses must be balanced by accessions of new alleles by mutation or outbreeding. If the values of $u, v$ or $m$, so calculated, 
do not agree with those originally assumed and these are large enough to affect appreciably the form of the distribution, it becomes necessary to try other values of $R$ until, by repeated trial and interpolation, agreement is reached.

\section{THE RELATIONS BETWEEN MUTATION RATE, POPULATION SIZE AND NUMBER OF ALLELES}

It has been shown previously (WRIGHT I93I) that the rate of loss of alleles is usually about half the frequency of the subterminal class, thus, $K=\frac{1}{2} f(\mathrm{I} / 2 N)=(\mathrm{I} / 4 N) \phi(\mathrm{I} / 2 N)$. In the present case, however, there may be such strong selection pressure tending to increase the frequency of rare alleles that this formula requires modification. It is indeed obvious that it is impossible to lose an allele if only three are present without extinction of the population. If in this case $q_{1}$ happens to fall very low at any time (population nearly Ioo percent $S_{2} S_{3}$ ), $q_{1}$ automatically rises almost to the opposite extreme $\left(q_{1}=.50\right)$ in the next generation. For larger values of $n, \Delta q$ may be taken as $\hat{q} q /(\mathrm{I}-3 \hat{q})$ where $q$ is small. As $\hat{q}$ is close to $\bar{q}(=\mathrm{I} / n)$ except in extreme cases, we may take $\Delta q=q /(n-3)$ as a rough approximation for small $q$ and $n>3$. The chance of loss with a given small value of $q$ is $e^{-2 N(q+\Delta q)}$ or $e^{-2 N q(n-2) /(n-3)}$ approximately, making allowance for selection. On testing different extreme types of distribution (such as $\phi(q)=\mathrm{I}, \phi(q)=C / q)$, it may be concluded that the fraction $(n-3) / 2(n-\mathrm{I})$ gives with sufficient accuracy for our present purpose, the portion of the subterminal class lost, (o if $n=3, \mathrm{I} / 6$ if $n=4, \frac{1}{4}$ if $n=5$, approaching the typical value $\frac{1}{2}$ as $n$ becomes large).

$$
K=\frac{(n-3)}{2(n-\mathrm{r})} f(\mathrm{I} / 2 N) \text { approximately. }
$$

Assume now that a limited number $\left(n^{\prime}\right)$ of alleles is possible, of which $n$ are present at any moment. The chance that any given allele, absent from the population, will arise in the next generation by mutation is $2 N v$, if $v$ is the rate of mutational origin of the allele in question. Assuming this to be the same for all of the $n^{\prime}$ alleles, the total number of missing alleles that will appear in a generation is $2 N\left(n^{\prime}-n\right) v$. Equating this to the number of losses, $K n$, we must have at equilibrium

$$
{ }_{2} N\left(n^{\prime}-n\right) v=\frac{n(n-3)}{2(n-\mathrm{I})} f(\mathrm{I} / 2 N) .
$$

If $u$ is the rate of mutation from one allele to all others, $u=\left(n^{\prime}-\mathrm{I}\right) v$

$$
u=\frac{n(n-3)\left(n^{\prime}-\mathrm{r}\right)}{4 N(n-\mathrm{I})\left(n^{\prime}-n\right)} f(\mathrm{I} / 2 N) .
$$


Figure I shows the number of alleles $(n)$ maintained in a population of size $N$ by various mutation rates $u$, assuming that 25 alleles are possible.

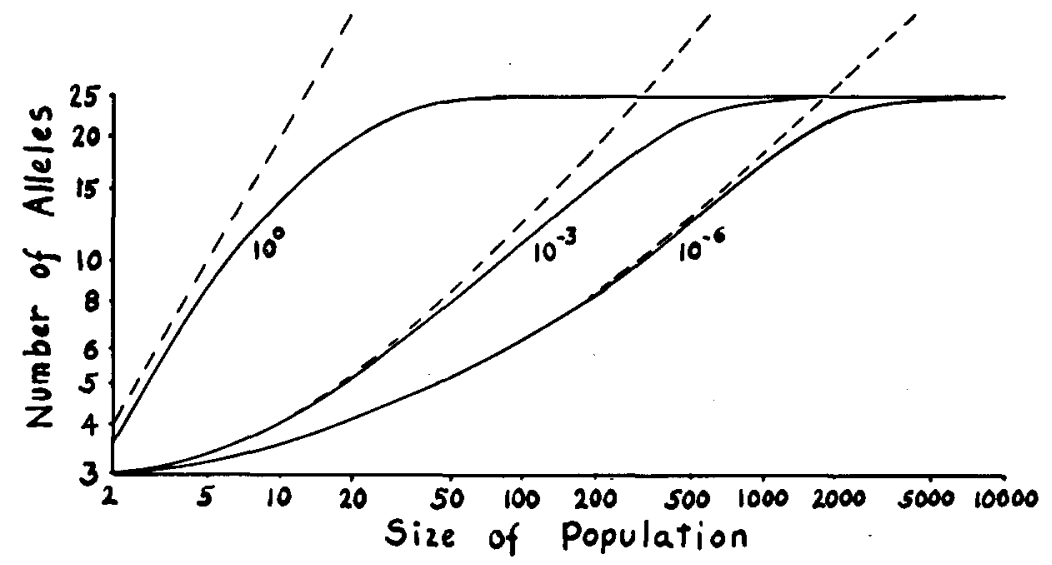

FIGURE I. - The number of self-sterility alleles maintained in populations of various sizes if ${ }_{25}$ different alleles are possible and mutation (or replacement by immigration) is occurring at the rates $10^{-3}$ or $10^{-6}$ per generation. The limiting case, complete replacement, is indicated by the curve labelled $10^{\circ}$. The broken lines show the number of alleles if an indefinitely large number are possible.

If new alleles are being introduced into a population to a significant extent by outbreeding rather than by mutation, corresponding equations can be written to express the balance of losses and gains. It is merely necessary to substitute $m q_{t}$ for $v, m\left(\mathrm{I}-q_{t}\right)$ for $u$ and $n_{t}\left(=\mathrm{I} / q_{t}\right)$, the number of alleles in the species as a whole, for $n^{\prime}$.

If the number of possible alleles is indefinitely great it is possible that each mutation will represent a wholly novel allele ( $v$ indefinitely small). In this case $\left(n^{\prime}-n\right) /\left(n^{\prime}-\mathrm{I}\right)$ approaches $\mathrm{I}$.

$$
u=\frac{n(n-3)}{4 N(n-\mathrm{I})} f(\mathrm{I} / 2 N) \text {. }
$$

Figure 2 shows the number of alleles $(n)$ maintained in a population of size $N$ by the occurrence of novel mutations at the rate $u$ per generation. Figures 3,4 and 5 compare the distributions of frequencies in populations of 50 and 500 individuals, at mutation rates of $10^{-2}, 1^{-4}$, and $10^{-6}$, respectively. The values of the selection index $R$, the mean number of representatives of an allele $(2 N \bar{q})$, the mean number of alleles $(n)$ and the turnover $(K)$ are given in table $\mathrm{r}$.

\section{COMPARISON WITH EMERSON'S DATA IN OENOTHERA ORGANENSIS}

In EMerson's data, 34 alleles were demonstrated in a sample of 135 gametes from a population estimated as less than 1000 plants and very 


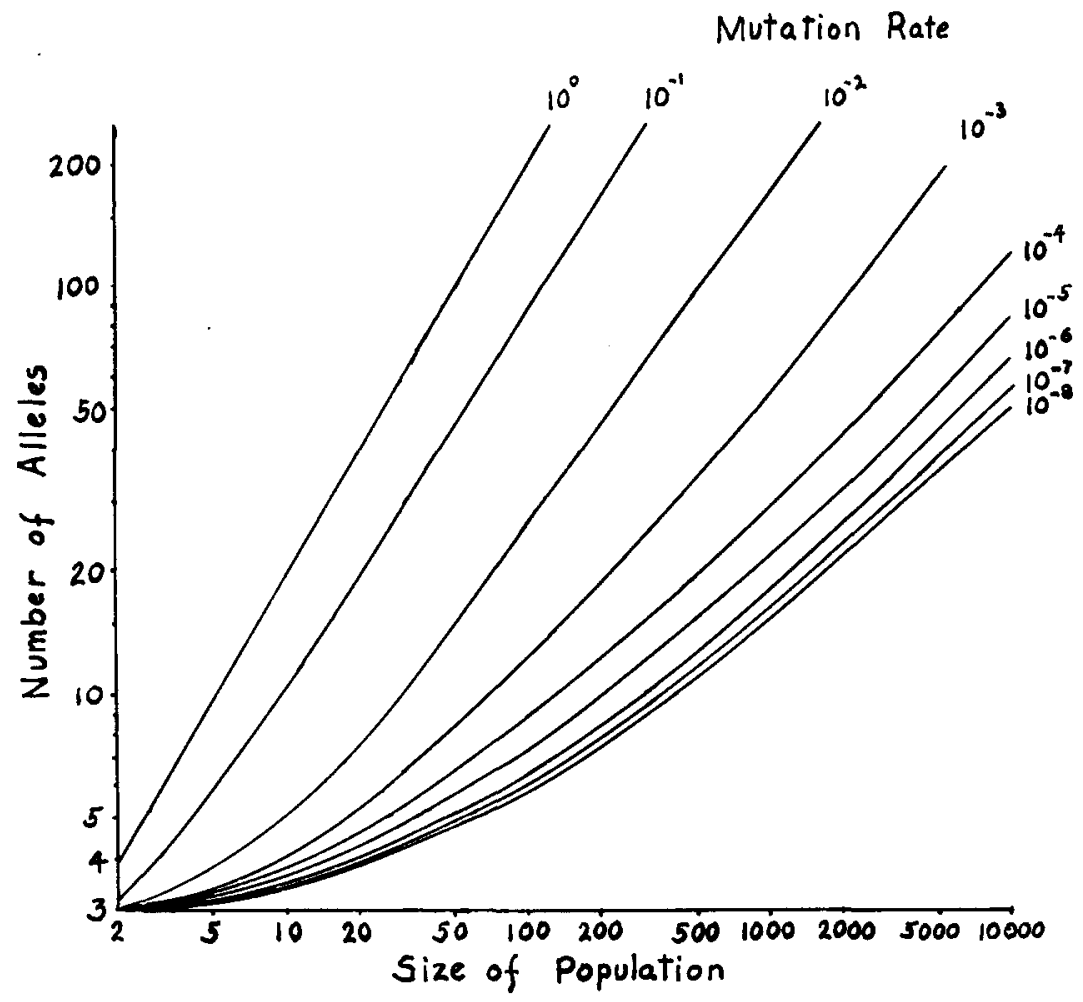

FIGURE 2.-The number of self-sterility alleles maintained in populations of various sizes if the number of possible alleles is indefinitely great and mutation (or replacement by immigration from a very large population) occurs at rates from $10^{\circ}$ to $10^{-8}$.

\section{TABLE I}

Comparison of populations of 50 and of 500 individuals at three different rates of mutation (or of immigration from an indefinitely large population).

\begin{tabular}{|c|c|c|c|c|c|}
\hline $\begin{array}{c}N \\
\text { No. OF } \\
\text { INDTVIDUALS }\end{array}$ & $\begin{array}{c}u \\
\text { MUTATION } \\
\text { RATE }\end{array}$ & $\begin{array}{c}R \\
\text { SELECTION } \\
\text { INDEX }\end{array}$ & $\begin{array}{c}2 N \bar{q} \\
\text { MEAN NO. OF } \\
\text { REPRESENTA- } \\
\text { TIVES OF ALLELE }\end{array}$ & $\begin{array}{c}n \\
\text { NO. OF } \\
\text { ALLELES }\end{array}$ & $\begin{array}{c}K \\
\text { FACTOR } \\
\text { TURNOVER } \\
\text { PERCENT }\end{array}$ \\
\hline 50 & .01 & .77 & 6.8 & 14.8 & 7 \\
\hline 500 & .01 & .95 & I0. 4 & 96.0 & Io \\
\hline 50 & $.000 \mathrm{r}$ & .59 & I 5.4 & 6.5 & 0.15 \\
\hline 500 & .0001 & .87 & $5 \mathrm{I} \cdot 3$ & 19.5 & $0.5 \mathrm{I}$ \\
\hline 50 & $.00000 \mathrm{I}$ & .49 & I9. 5 & $5 \cdot \mathrm{I}$ & 0.002 \\
\hline 500 & .000001 & .82 & $77 \cdot 7$ & 12.9 & 0.008 \\
\hline
\end{tabular}




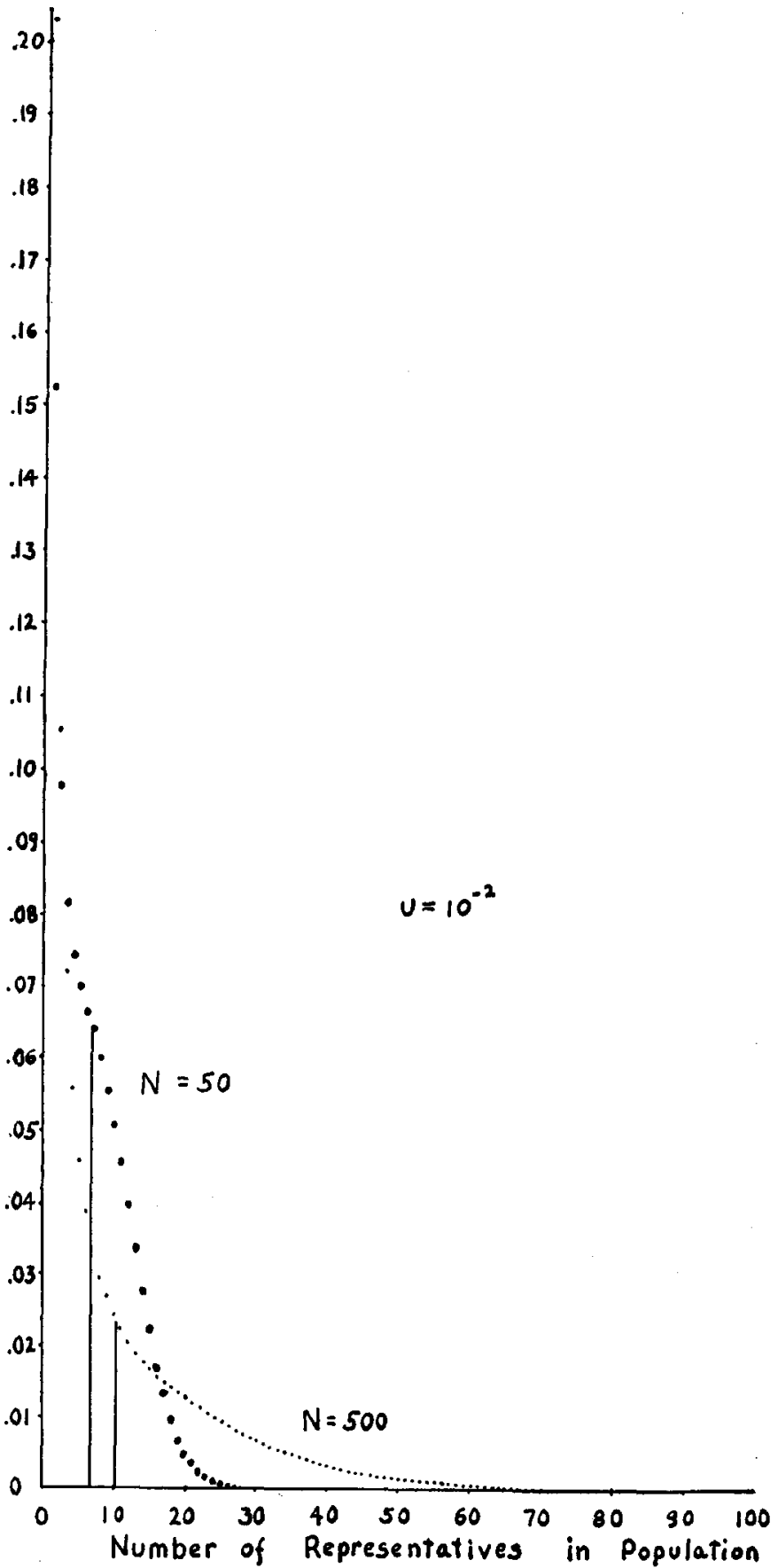

FIGURE 3.-Description under figures 4 and 5. 


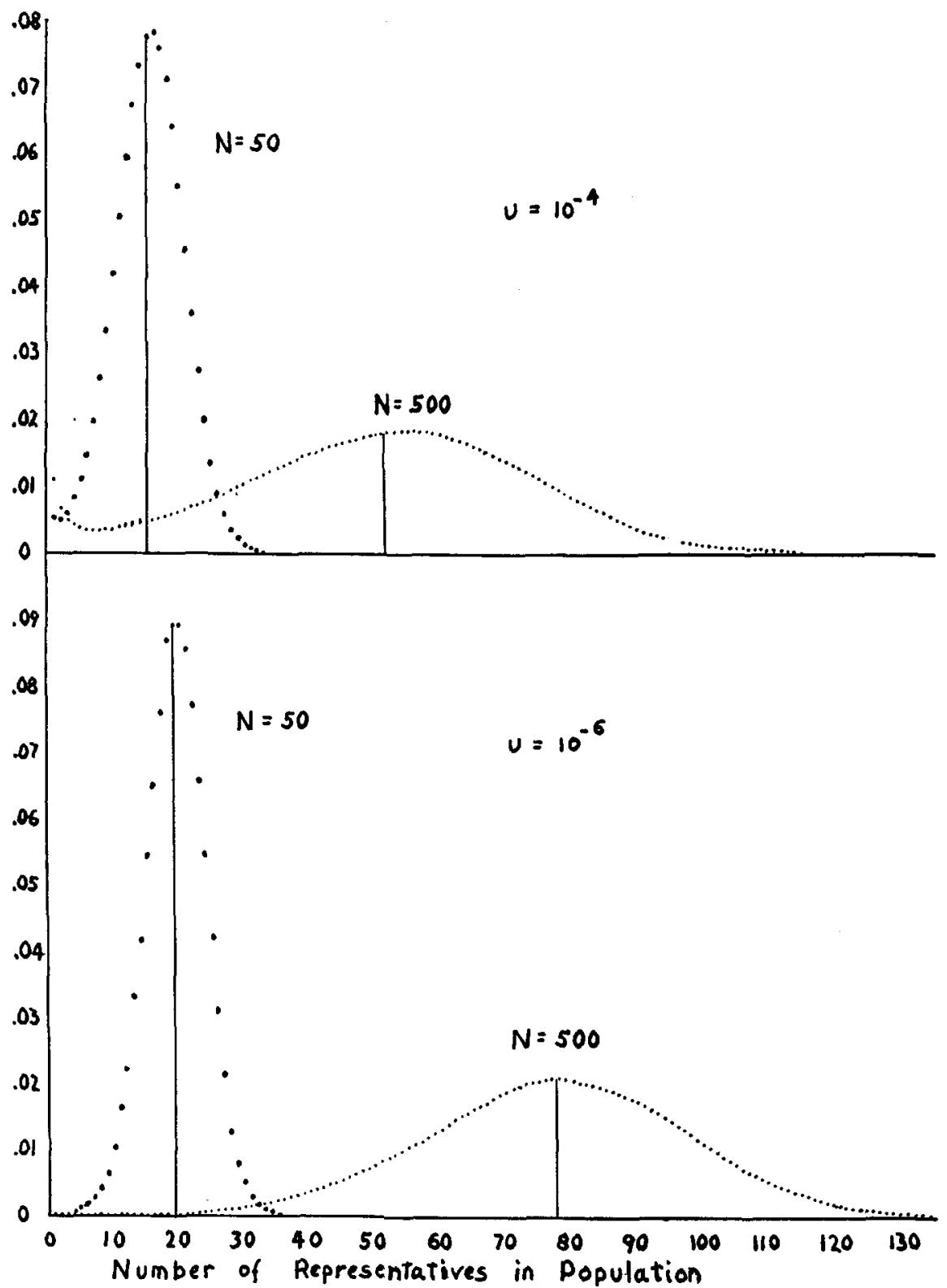

Figures 3 to 5.-The probabilities of different numbers of representatives $(2 N q)$ of a selfsterility gene (while present at all) compared in populations of 50 and 500 individuals and with mutation (or replacement by immigration from a very large population) at rates of $u=10^{-2}$ (fig. 3), $u=10^{-4}$ (fig. 4, above) or $u=10^{-6}$ (fig. 5, below). An indefinitely large number of alleles is assumed possible. See table I. 
likely less than 500 . It is known that several additional alleles are present in the population and doubtless the number would be considerably increased in a sample of size $2 N$. From figure 2, it is apparent that it would require a mutation rate of considerably more than $\mathrm{IO}^{-3}$ to maintain such a large number of alleles. Mutation rates of $1 \mathrm{r}^{-5}$ or less would maintain less than 15 alleles. Professor EMerson informs me that in preliminary tests no mutations have been found in 45,000 pollen grains, indicative as far as it goes of a mutation rate decidedly too low to account for the observed number of alleles.

One possible explanation would be that the size of the population is much greater than estimated from the data now at hand or, if not greater now, that it has recently been much greater, with loss of alleles at too slow a rate to have reached equilibrium. Inspection of figure 2 indicates that a population of some 4000 to 5000 is required to account for the probable number of alleles. Another possible explanation would be that some alleles are much more mutable than others. An average rate greater than $10^{-3}$ seems, however, improbable. Finally, there is the possibility that the large number of alleles is a consequence of local inbreeding. This possibility may be considered by extension of the methods used here.

\section{THE EFFECT OF LOCAL INBREEDING}

It is obvious from inspection of figure 2 that subdivision into completely isolated groups would greatly increase the number of alleles carried by the species as a whole, although reducing the number found in a single local collection. In the long run each group would come to have an entirely different set of alleles from every other group. As an isolated population of 50 individuals would maintain five or six alleles at mutation rates of Io $^{-6}$ or $10^{-5}$, an assemblage of 10 such groups would maintain 50 or 60 alleles instead of the $I_{3}$ to 15 expected under random mating. With a finer subdivision, the increase would be still greater.

However, it is clear from Emerson's data that there is nothing approaching complete isolation. Of the 34 alleles found in the 1937 collection, seven were found in all three of the most thoroughly studied canyon populations, I4 were found in two of them, leaving $\mathrm{I} 3$ which have so far been found in only one. On the other hand, as he points out, there is not completely random interbreeding. There is almost twice as much chance (II4 $/ 2699=.043$ ) that a second gamete from the same canyon as the one chosen first will have the same allele, as that one from another canyon will agree $(\mathrm{I} 28 / 545 \mathrm{I}=.024)$. Even the slightest restriction on random breeding will presumably increase the number of alleles carried by the total population, but whether this increase is an appreciable one where there is as much interbreeding as is indicated in the present case, requires consideration. 
We have already considered the distribution of gene frequencies in a local population of size $N$, receiving the proportion $m$ of its gametes from the species as a whole, with gene frequency $q_{t}$. If the species is not indefinitely large, $q_{t}$ itself is a variable and it is, indeed, the distribution of $q_{t}$ which we wish ultimately to estimate. However, $q_{t}$ enters into the formula for $\phi(q)$ in such a way that there is no important error in assuming it constant at the value $\bar{q}_{t}=\mathrm{I} / n_{t}$ where $n_{t}$ is the total number of alleles at which we wish to aim in our calculations. The value of $R$ for the local population may be found as before by trial and interpolation to satisfy the condition of equilibrium.

In considering the species as a whole, it is convenient first to distinguish the proportion $\mathrm{I}-2 m$ of the fertilizations in which the pollen as well as the ovules come from the local group and the proportion $2 m$ in which the pollen is a random sample from the species. In the former, selection pressure depends on the number of alleles in the group and hence on the value of $R$ determined for the group. In the latter it depends on the number of alleles in the whole species. We will consider first the component due to inbreeding.

Assume that there are $G$ subgroups of equal size $(N)$. Let $h$ be the proportion of these that contain the allele $S_{i}$. Then $q_{t} / h$ is the average frequency within these groups. $S_{i}$ pollen has no chance of success on the $2 q_{t}$ plants that carry $S_{i}$. On the $\left(h-2 q_{t}\right)$ plants that are of the same subgroups but do not carry $S_{i}$, the average frequency of success of $S_{i}$ pollen to other kinds is in the ratio $q_{t} / h: R\left[\mathrm{I}-\left(q_{t} / h\right)\right]$. The frequency of functioning $S_{i}$ pollen grains is thus

$$
\frac{\left(h-2 q_{t}\right) q_{t} / h}{q_{t} / h+R\left[\mathrm{I}-\left(q_{t} / h\right)\right]}
$$

and the frequency of functioning $S_{i}$ gametes is

$$
\frac{q_{t}}{2}\left[\frac{\left(h-q_{t}\right)(\mathrm{I}+R)}{R h+q_{\iota}(\mathrm{I}-R)}\right] .
$$

It will not do even as a first approximation, to assume that $h$ is constant. If the allele is represented only once in the whole species $\left(q_{t}=1 / 2 N_{t}\right), h$ is obviously $\mathrm{I} / G$. With increasing representation in the species, $h$ may be expected to increase until a point is reached at which the gene is practically always present in all subgroups $(h=\mathrm{I})$. As a first approximation, we will assume that $h$ varies linearly with $q_{t}$ between $q_{t}=1 / 2 N_{t}$ and $h=\mathrm{I}$, with such a slope $(b)$ as will yield the required $\bar{q}_{t}$.

$$
h=\frac{\mathrm{I}}{G}+b\left(q_{t}-\frac{\mathrm{I}}{2 N_{t}}\right) .
$$


It will be convenient to write this, $h=a+b q_{t}$, where $a=\left(\mathrm{I} / G-b / 2 N_{t}\right)$.

In those fertilizations which may be treated as random breeding, the frequency of functioning $S_{i}$ gametes is

$$
\frac{q_{t}}{2} \frac{\left(\mathrm{I}-q_{t}\right)\left(\mathrm{I}+R_{t}\right)}{\left[R_{t}+q_{t}\left(\mathrm{I}-R_{t}\right]\right.}
$$

where $R_{t}$ depends on the number of alleles in the whole species. If the amount of crossbreeding is small, this term is relatively unimportant and a rough approximation will suffice. Indeed if $m=$. or or less, it makes little difference whether this term is considered, or whether it is omitted altogether, giving full weight to the inbreeding term. If included, we may use $R_{t}=\left(n_{t}-3\right) /\left(n_{t}-\mathrm{I}\right)$, the value if all alleles are equally frequent, as such an approximation. Giving inbreeding and crossbreeding due weight and introducing mutation pressure, the rate of change of gene frequency may be written

$$
\begin{aligned}
\Delta q_{t}= & (\mathrm{I}-2 m) \frac{(\mathrm{I}+R)}{2} q_{t}\left[\frac{a+q_{t}(b-\mathrm{I})}{R a+q_{t}(R b+\mathrm{I}-R)}\right] \\
& +2 m \frac{\left(\mathrm{I}+R_{t}\right)}{2} q_{t}\left[\frac{\mathrm{I}-q_{t}}{R_{t}+q_{t}\left(\mathrm{I}-R_{t}\right)}\right]-q_{t}(\mathrm{I}+u) .
\end{aligned}
$$

The sampling variance of a subgroup is $q(\mathrm{r}-q) / 2 N$ disregarding crossbreeding. That of the whole species is

$$
\sigma_{\Delta q_{i}}^{2}=\frac{\mathrm{I}}{G^{2}} \Sigma\left(\frac{q(\mathrm{I}-q)}{2 N}\right)
$$

noting that $\Delta q_{t}=\Sigma \Delta q / G$. A first approximation can be obtained by replacing $q$ by its average value $\left(q_{t} / h\right)$ in those groups ( $h G$ in number) that contain it at all.

$$
\sigma_{\Delta q_{t}}^{2}=\frac{q_{t}\left(h-q_{t}\right)}{{ }_{2} N_{t} h}=\frac{q_{t}\left[a+q_{t}(b-\mathrm{r})\right]}{2 N_{t}\left(a+b q_{t}\right)} \text { approximately. }
$$

If $q_{t}$ is small, this differs little from the value $q_{t}\left(\mathrm{I}-q_{t}\right) / 2 N_{t}$ in a random breeding population of the same size. The latter may be used for convenience in dealing with the small crossbreeding term in the ratio $\Delta q_{t} / \sigma_{\Delta q_{t}}^{2}$.

Substitution in the general formula for $\phi(q)$ leads to the following expression.

$$
\begin{aligned}
\log _{10} \phi\left(q_{t}\right)= & \log _{10}\left(a+b q_{t}\right)-\log _{10} q_{t} \\
& +\left[\frac{4 N_{t} a(\mathrm{I}+u)}{(b-\mathrm{I})^{2}}-\mathrm{I}\right] \log _{10}\left[a+q_{t}(b-\mathrm{I})\right] \\
& +\left[\log _{10} e\right]\left[\frac{2 N_{t} b(\mathrm{I}+R)(\mathrm{I}-2 m)}{\mathrm{I}+R(b-\mathrm{I})}-\frac{4 N b(\mathrm{I}+u)}{b-\mathrm{I}}\right] q_{t}
\end{aligned}
$$




$$
\begin{aligned}
& +(\mathrm{I}-2 m) \frac{2 N_{t} a\left(\mathrm{I}-R^{2}\right)}{[\mathrm{I}+R(b-\mathrm{I})]^{2}} \log _{10}\left[R a+q_{t}[\mathrm{I}+R(b-\mathrm{I})]\right. \\
& +{ }_{4} m N_{t} \frac{\left(\mathrm{I}+R_{t}\right)}{\left(\mathrm{I}-R_{t}\right)} \log _{10}\left[R_{t}+q_{t}\left(\mathrm{I}-R_{t}\right)\right]
\end{aligned}
$$

Values of $b$ and $u$ may be found by trial that give the required mean $\left(\bar{q}_{t}\right)$ and satisfy the balance between loss and mutation.

Four cases have been worked through to obtain an indication of the effect of subdivision into partially isolated populations (table 2 ). In the first case, a total population of 500 was assumed to be divided into ro equal

TABLE 2

Comparison of subdivided and random breeding populations of 500 individuals and of the partially isolated groups of the former. The columns $N, m$ and $u$ describe the conditions with respect to size of population, immigration and mutation. Columns $R$ and $b$ give two additional constants of the formulae. Column $n$ gives the number of alleles maintained under the given conditions and column $K$

\begin{tabular}{|c|c|c|c|c|c|c|c|}
\hline & $\begin{array}{c}N \\
\text { No. OF } \\
\text { INDI- } \\
\text { VIDUALS }\end{array}$ & $\begin{array}{c}m \\
\text { IMMIGRA- } \\
\text { TION } \\
\text { INDEX }\end{array}$ & $\begin{array}{c}u \\
\text { MUTA- } \\
\text { TION } \\
\text { RATE }\end{array}$ & $\begin{array}{c}R \\
\text { SELEC- } \\
\text { TION } \\
\text { INDEX }\end{array}$ & $b$ & $\begin{array}{c}n \\
\text { No. OF } \\
\text { ALLELES }\end{array}$ & $\begin{array}{c}K \\
\text { FACTOR } \\
\text { TURNOVER } \\
\text { PERCENT }\end{array}$ \\
\hline Group & $5^{\circ}$ & .or & .000045 & $.73^{2}$ & 一 & II. 6 & 3.6 \\
\hline Total & 500 & o & .000045 & $.73^{2}$ & 6.73 & 20.0 & 0.23 \\
\hline Random & 500 & o & .000045 & .860 & - & 17.5 & 0.25 \\
\hline Group & 50 & $.0 \mathrm{I}$ & .0010 & .755 & - & 13.4 & $5 \cdot 3$ \\
\hline Total & 500 & $\circ$ & .0010 & .755 & 5.29 & 50.0 & 2.0 \\
\hline Random & 500 & $\circ$ & .0010 & .906 & - & 33.8 & 3.0 \\
\hline Group & 50 & .001 & .00020 & $.65^{8}$ & - & 8.2 & 1.0 \\
\hline Total & 500 & $\circ$ & .00020 & $.65^{8}$ & 2.50 & 50.0 & 0.43 \\
\hline Random & 500 & $\circ$ & .00020 & .880 & - & 22.1 & 0.90 \\
\hline Group & 10 & .01 & .00009 & .425 & - & $5 \cdot 3$ & $3 \cdot 3$ \\
\hline Total & 500 & 0 & .00009 & .425 & $3 \cdot 73$ & 50.0 & 0.18 \\
\hline Random & 500 & 0 & .00009 & .869 & - & 19.2 & 0.45 \\
\hline
\end{tabular}
the percentage turnover of alleles per generation.

groups breeding within themselves except for 2 percent foreign pollen. It is required to find the mutation rate that will maintain 20 alleles in the species. By trial it was found that the value of $R$ within groups is .732 and that the mean number of alleles within groups is II. 6 with turnover of 3.6 percent per generation. For the whole population, $b$ was found to be 6.73 and the required mutation rate $4.5 \times 10^{-5}$. The rate of turnover of alleles in the species is 0.23 percent. In a completely random breeding population, the same mutation rate would maintain an average of 17.5 alleles with a turnover of 0.25 percent per generation. In this case the 


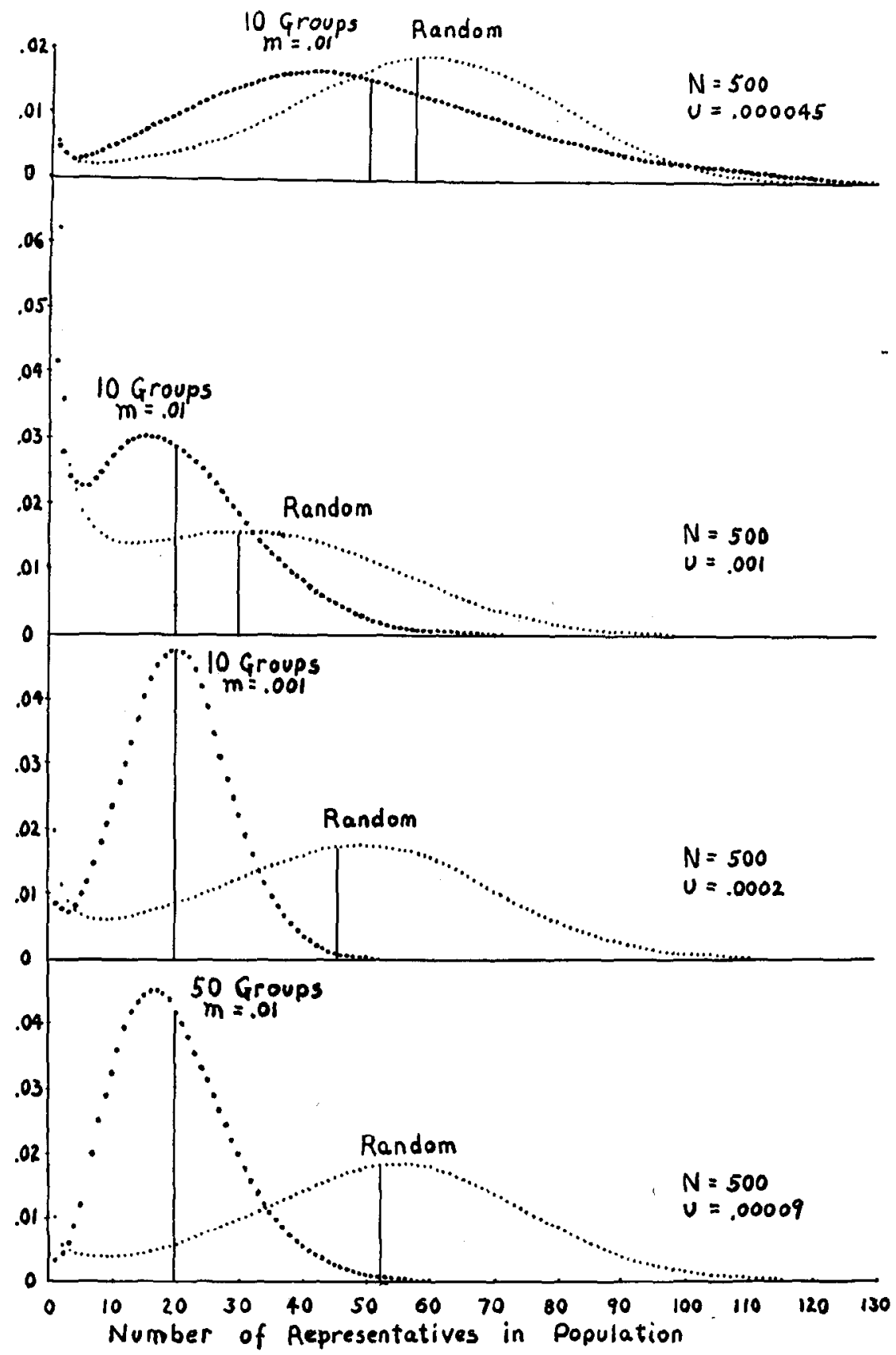

FIgUREs 6-9. (See opposite page for description.) 
postulated amount of subdivision increases the number of alleles only about 14 percent. The distribution in the subdivided and random breeding populations are compared in figure 6 .

With the same amount of subdivision ( $G=$ Io, $N=5 \circ, m=.0$ I) it requires a mutation rate of about $\mathrm{Io}^{-3}$ to maintain 50 alleles (turnover 2.0 percent per generation). This mutation rate would maintain only about 34 alleles under random mating. The subgroups carry I3.4 alleles on the average (figure 7 ).

It seemed of interest next to investigate the effect of more complete isolation. It was again assumed that there are ro subgroups of 50 individuals each but the crossbreeding index was taken as .OOI instead of .OI. It only requires a mutation rate of $2 \times \mathrm{IO}^{-4}$ to maintain 50 alleles in the species (turn over 0.4 percent), a mutation rate that would maintain 22 alleles under random mating. The subgroups carry only 8.2 alleles on the average (figure 8 ).

None of the preceding cases approaches a satisfactory interpretation of the situation described by Emerson in Oenothera organensis. To maintain the probable number of alleles in the species requires a higher mutation rate and more extreme isolation than is probable. The subgroups postulated in all of these cases (5o individuals) are roughly comparable to the populations of separate canyons. More alleles have been observed in the separate canyons (average for the three most studied, 20.7) than is permitted by the theory (about eight in the last case).

The only possible interpretation, accepting 500 as an estimate of the total population, assuming mutation rates less than $10^{-4}$ and assuming equilibrium, seems to be along the line of a finer subdivision. It was accordingly assumed next that the species is divided into 5o local groups of Io individuals and that these groups breed within themselves except for 2 percent foreign pollen. With this degree of subdivision, it requires a mutation rate of $9 \times 10^{-5}$ to maintain 50 alleles in the species (turnover O.I8 percent). This rate would maintain only about ig alleles under random

\section{Explanation of Figures 6-9}

Frgures 6-9.-(from top to bottom). The probabilities of different numbers of representatives of a self-sterility allele (while present at all) in populations of 500 individuals, compared in the cases of random breeding and of subdivision into partially isolated groups.

Figure 6.-Ten groups, $m=. \mathrm{or}$, 20 alleles in the subdivided population. The implied mutation rate $\left(4.5 \times 10^{-5}\right)$ would maintain 17.5 alleles under random mating.

FIGURE 7.-Ten groups, $m=$.or, 50 alleles in the subdivided population. The implied mutation rate $\left(\mathrm{IO}^{-3}\right)$ would maintain 34 alleles under random mating.

Figure 8.-Ten groups, $m=. \infty \mathrm{I}, 50$ alleles in the subdivided population. The implied mutation rate $\left(2 \times 10^{-4}\right)$ would maintain 22 alleles under random mating.

FIGURE 9.-Fifty groups, $m=.0 \mathrm{r}, 50$ alleles in the subdivided population. The implied mutation rate $\left(9 \times 10^{-5}\right)$ would maintain ${ }_{9} 9$ alleles under random mating. 
mating (figure 9). The groups of ro plants should carry an average of only 5.3 alleles; but a canyon containing several such groups, would, of course, maintain several times this number.

This last case obviously comes closest to a satisfactory interpretation and even closer inbreeding would be still more satisfactory. It appears that if plants are pollinated in some 98 percent or more of the cases by their immediate neighbors and only 2 percent or less by a random sample of pollen from the species as a whole, it would be possible for a species of only 500 individuals to maintain 40 or 50 alleles by mutation rates of the order of $10^{-5}$ or $10^{-6}$ per generation.

\section{LITERATURE CITED}

EAST, E. M., and MANGELSDORF, A. J., I925 A new interpretation of the hereditary behavior of self-sterile plants. Proc. Nat. Acad. Sci. II: 166-17I.

EMERson, S., 1938 The genetics of self-incompatibility in Oenothera organensis. Genetics 23: I90-202.

I939 A preliminary survey of the Oenothera organensis population. Genetics 24: 524-537.

WRIGHT, S., I93 I Evolution in Mendelian populations. Genetics I6: 97-I59.

I938 The distribution of gene frequencies under irreversible mutation. Proc. Nat. Acad. Sci. 24: $253^{-259}$. 